\title{
Effects of Sitagliptin on the Coronary Flow Reserve, Circulating Endothelial Progenitor Cells and Stromal Cell-derived Factor-1alpha
}

\author{
Tetsuji Morishita, Hiroyasu Uzui, Hiroyuki Ikeda, Naoki Amaya, Kenichi Kaseno, \\ Kentaro Ishida, Yoshitomo Fukuoka and Hiroshi Tada
}

\begin{abstract}
:
Objective Circulating endothelial progenitor cells (EPCs) are regulated by stromal cell-derived factor-1alpha (SDF-1 $\alpha)$ and are reduced in type 2 diabetes mellitus (DM). SDF- $1 \alpha$ is a substrate of dipeptidylpeptidase-4 (DPP-4), so we investigated whether or not DPP-4-inhibitors modulate EPC levels in type 2 DM patients with coronary artery disease (CAD).

Methods Thirty patients with CAD and type 2 DM treated using an ordinary regimen were enrolled. EPC and SDF- $1 \alpha$ levels were compared between those receiving additional 24-week treatment with a DPP-4inhibitor $(\mathrm{n}=11)$ and no additional treatment $(\mathrm{n}=19)$. We determined the HbA1c, 1.5-Anhydro-D-glucitol $(1,5-$ AG), coronary flow reserve (CFR), brain natriuretic peptide (BNP), E/e', and circulating EPC proportion and SDF- $1 \alpha$ levels at baseline and the end of follow-up. The CFR was assessed using a dual-sensor-equipped guidewire. The primary endpoints were changes in the EPC count, SDF-1 $\alpha$ levels, and CFR from baseline to the end of follow-up. The secondary endpoints were changes in the HbAlc and 1,5-AG, which are useful clinical markers of postprandial hyperglycemia, as well as the BNP and E/e'.

Results After the 6-month follow-up, compared with ordinary regimen subjects, the patients receiving a DPP-4-inhibitor showed no significant increase in the EPC proportion $(-0.01 \pm 0.50$ vs. $0.02 \pm 0.77 \%, \mathrm{p}=0.87)$, SDF- $1 \alpha$ level $(-600.4 \pm 653.6$ vs. $-283.2 \pm 543.1 \mathrm{pg} / \mathrm{mL}, \mathrm{p}=0.18)$, or CFR $(0.0 \pm 0.2$ vs. $0.1 \pm 0.6, \mathrm{p}=0.20)$, whereas both the 1.5 -AG level $(2.4 \pm 4.6$ vs. $-0.7 \pm 2.5 \mu \mathrm{g} / \mathrm{dL}, \mathrm{p}=0.07)$ and $\mathrm{HbA1c}(-0.8 \pm 1.8$ vs. $0.0 \pm 0.7 \%, \mathrm{p}=$ 0.02 ) were improved. There were no significant differences between the two groups in changes in the BNP and E/e'.

Conclusion DPP-4 inhibition with sitagliptin did not increase or decrease the EPC proportion, SDF-1 $\alpha$ level, or CFR, although the glycemic control was improved.
\end{abstract}

Key words: endothelial progenitor cell, stromal cell-derived factor-1, dipeptidyl-peptidase-4

(Intern Med 58: 2773-2781, 2019)

(DOI: 10.2169/internalmedicine.2616-19)

\section{Introduction}

Circulating endothelial progenitor cells (EPCs) have the ability to differentiate into mature endothelial cells and can elicit angiogenesis, vasculogenesis (1), and vessel repair in cardiac ischemia $(2,3)$, vascular injuries, and diabetic vasculopathy caused by endothelial damage (4). EPCs play pivotal roles in atherosclerosis, myocardial ischemia, and revas- cularization. However, the regulation of EPC recruitment to ischemic tissues is an issue.

EPCs are regulated by several factors, including vascular endothelial growth factor (5) and stromal cell-derived factor1 alpha (SDF-1 $\alpha)(6)$. SDF-1 $\alpha$ is a chemokine considered to attract EPCs to ischemic tissues and increase the migratory function from bone marrow to peripheral blood (7). The administration of SDF- $1 \alpha$ attenuates EPC apoptosis and augments EPC-induced vasculogenesis (7), resulting in im- 
proved ischemic tissue perfusion. SDF- $1 \alpha$ is a substrate of dipeptidyl-peptidase-4 (DPP-4), which cleaves and degrades SDF-1. Decreased DPP-4 activity is thus considered to upregulate the number of EPCs in peripheral blood (8).

One pioneering work reported that the addition of a DPP4 inhibitor significantly increased EPC counts and SDF- $1 \alpha$ levels (9). The administration of a DPP-4 inhibitor is therefore likely to contribute to an improved vascular function (10-12) via the upregulation of SDF- $1 \alpha$ and EPCs.

Sitagliptin is a widely used oral antidiabetic agent known to be effective in managing the diabetic metabolism. However, little is known about its effect on the coronary microcirculation and atherosclerosis (13). We therefore conducted a prospective, two-group, parallel-design study to assess the effects of DPP-4 inhibitor on the EPC count, SDF-1 $\alpha$ levels, and coronary flow reserve (CFR) in patients with type 2 diabetes mellitus (DM) and coronary artery disease (CAD). The CFR, measured using a Doppler guidewire, represents the coronary microvascular function, and a reduced CFR $(<2.0)$ is considered to indicate coronary microvascular dysfunction (14). Along with the coronary endothelial function, levels of asymmetric dimethylarginine (ADMA), a circulating endogenous nitric oxide synthase inhibitor $(15,16)$, were also measured in order to ascertain whether or not DPP-4 inhibitor therapy influenced the systemic endothelial function. The effect of incretin-based therapy on increasing heart failure has recently garnered attention (17). To test whether or not the cardiac function was affected by DPP-4 inhibitor therapy, we analyzed the changes in the brain natriuretic protein (BNP) level and the ratio between the early mitral inflow velocity and mitral annular early diastolic velocity (E/e') between the two groups.

It has been reported that DPP-4 inhibitors exert antiatherogenic effects in animal models, whereas DPP-4 inhibitors did not appear to decrease the number of major adverse cardiovascular events in large clinical trials. Thus, the effects of DPP-4 inhibitors on cardiovascular events and atherosclerosis remain controversial, and little is known about the effects of these drugs, particularly on coronary microcirculation. Therefore, we examined the effects of the DPP-4 inhibitor sitagliptin on the CFR using a Doppler guidewire as the primary endpoint. The purpose of our study was to explore the impact of DPP-4 inhibitors on coronary microcirculation, and the strength of our study was that the actual intra-coronary hemodynamics were measured using an intracoronary Doppler guidewire.

\section{Materials and Methods}

\section{Study subjects and design}

Patients were recruited from the Department of Cardiovascular Medicine of the University of Fukui Hospital. Patients were eligible for inclusion if they had CAD with significant stenosis on coronary angiography and had received percutaneous coronary intervention (PCI). CAD was defined as coronary artery stenosis with $>50 \%$ diameter stenosis confirmed by invasive angiography. To evaluate the association with EPC proportions, peripheral EPCs were assessed as CD34+ cells co-expressing both CD133 and vascular endothelial growth factor receptor- 2 in $30 \mathrm{CAD}$ patients (mean age, 69.2 \pm 11.3 years old) with DM. Serum 1,5-AG and hemoglobin $(\mathrm{Hb}) \mathrm{A} 1 \mathrm{c}$ levels were also measured. EPC counts and SDF-1 $\alpha$ levels were compared between patients receiving additional 24-week treatment with a DPP-4 inhibitor ( $\mathrm{n}=$ 11) and those with no additional treatment $(n=19)$. The DPP-4 inhibitor group received sitagliptin at $50 \mathrm{mg}$ once daily. Attending physicians were free to choose the pharmacotherapy for control of DM. We determined the HbA1c, 1,5-anhydro-D-glucitol (1,5-AG) level, proportion of circulating EPCs, and plasma concentrations of SDF-1.

The effect of six months of treatment on the CFR was assessed in non-culprit vessels. Angiographic follow-up was scheduled for all patients six months after PCI. At the sixmonth follow-up, repeated CFR measurements in the nonculprit vessel were performed. All PCI procedures were performed with stent placement, and post-PCI management was performed in a standard manner.

Ethics committee approval was obtained, and all patients gave their written informed consent before participation. Patients with a history of neoplastic, hepatic, infectious, or autoimmune diseases were excluded from this study. Patients receiving acarbose were also excluded, as this $\alpha$ glucosidase inhibitor may potentially reduce 1,5-AG levels by interfering with the intestinal absorption of 1,5-AG (18).

Whole blood was withdrawn from a forearm or femoral vein and kept on ice. Plasma and serum samples were separated by centrifugation within 30 minutes. After centrifugation, samples were frozen and stored at $-80^{\circ} \mathrm{C}$ until use.

\section{Definition of risk factors}

The studied population was screened for cardiovascular risk factors. The definitions of hypertension, DM, dyslipidemia, and obesity have been previously reported (19).

\section{Flow cytometric analyses}

EPCs were quantified in collected blood samples by flow cytometry and analyzed for the expression of CD34, CD45, CD133, and vascular endothelial growth factor receptor-2 with four-color flow cytometry (FACSCalibur; BD Biosciences, Franklin Lakes, USA). For more details on this procedure, please see the previous report (20).

EPC values were defined as the percentage of CD34+, CD45dim, CD133+, and vascular endothelial growth factor receptor-2+ cells per CD34+CD45dim cell fraction, as described previously (20). To identify small populations of EPC fractions, at least 2,000,000 events were measured with CD45+ gating. The coefficient of variation was less than $5 \%$.

\section{Determination of serum 1,5-AG and HbA1c}

Levels of 1,5-AG were measured using an enzymatic as- 
say with a two-reagent system (Determiner L 1,5-AG; Kyowa Medex, Tokyo, Japan) according to the instructions from the manufacturer. HbA1c was assayed by highperformance liquid chromatography. Postprandial hyperglycemia was defined as a serum 1,5-AG level $<14.0 \mu \mathrm{g} /$ $\mathrm{mL}(21,22)$.

\section{Measurement of plasma BNP}

Plasma levels of BNP were measured using a commercially available radioimmunoassay kit (Shionogi, Osaka, Japan) (23).

\section{SDF-1 $\alpha$ and ADMA measurements}

Measurements of SDF- $1 \alpha$ and ADMA levels were performed using commercially available sandwich enzyme linked immunosorbent assays for SDF-1 $\alpha$ (R\&D Systems, Minneapolis, USA) and ADMA (Perseus Proteomics, Tokyo, Japan), respectively (20).

\section{Echocardiography}

The left ventricular (LV) ejection fraction (EF) was estimated by Simpson's modified two-dimensional biplane method from four- and two-apical chamber views (24). Tissue Doppler measurements were performed to assess the LV diastolic function and mitral annulus moving speed waveform. The ratio of the LV inflow blood flow early diastolic wave (E) to the early diastolic mitral annulus velocity wave movement (e') was used as an indicator of the LV diastolic function (25).

\section{Coronary angiography and the evaluation of CFR}

An arterial cannula was placed in the radial or femoral artery. Coronary angiography was then performed after the administration of intracoronary isosorbide dinitrate to confirm the absence of significant epicardial coronary artery stenosis in the reference vessel. Aortic pressure $(\mathrm{Pa})$ was measured through the guiding catheter. After coronary angiography, the pressure $(\mathrm{Pd})$ and flow velocity distal to the reference site were measured simultaneously with a 0.014-inch dualsensor (pressure and Doppler velocity) guidewire (ComboWire, Volcano Therapeutics, Rancho Cordova, USA). After calibration, advancement through the catheter, and equalization with $\mathrm{Pa}$ in the catheter, the wire was placed at least 3-4 cm distal to the segment of each coronary artery and manipulated until an optimal and stable velocity signal was obtained. The position of the tip of the wire was confirmed by fluoroscopy and angiography. The $\mathrm{Pa}, \mathrm{Pd}$, instantaneous peak velocity, and electrocardiogram were obtained online at baseline and after the induction of maximal hyperemia with an intracoronary bolus injection of $50 \mu \mathrm{g}$ of adenosine triphosphate (ATP) in the left coronary artery (LCA) and an intracoronary bolus injection of $30 \mu \mathrm{g}$ of ATP in the right coronary artery (RCA) (ComboMap Pressure and Flow System; Volcano Therapeutics). The fractional flow reserve (FFR) was calculated as the ratio of the mean $\mathrm{Pd}$ to the mean Pa during maximal hyperemia. The CFR was calculated as the ratio of the time-averaged peak hyperemic to the baseline average flow velocity. Intracoronary ATP (LCA: $50 \mu \mathrm{g}$; RCA: $30 \mu \mathrm{g}$ ) was administered until maximal hyperemia had been achieved in order to calculate the ATP-induced CFR. CFR ratios $<2.0$ were considered to indicate coronary microvascular dysfunction $(14,26,27)$.

\section{Statistical analyses}

Continuous values are expressed as medians (interquartile range) or mean \pm standard deviation, as appropriate. The chi-square test was used to compare categorical data. Comparisons between two groups of continuous measurements were performed using Mann-Whitney U tests. Correlations between two variables were assessed by Spearman's rank test.

Univariate and multivariate linear regression analyses were performed to identify predictors of the $\triangle \mathrm{EPC}$ (The mean change from baseline in the EPC proportion). The sample size was calculated as described in a previous study evaluating the effect of sitagliptin on EPCs (9) because of the limited information available on the effect of DPP-4 inhibitors on EPCs. Based on the assumption that EPCs increased by $1.0 \%$ difference in the DPP-4 inhibitor group with a standard deviation of $0.6 \%$ between the sitagliptintreated and no additional treatment groups, a total of 14 subjects would be required at a 5\% significance level and $80 \%$ power. On the assumption that the CFR increased by 0.7 difference in the DPP-4 inhibitor group with a standard deviation of 0.4 between the sitagliptin-treated and no additional treatment groups, a total of 10 subjects would be required at a $5 \%$ significance level and $80 \%$ power. On the assumption that SDF- $1 \alpha$ increased by a $1,000 \mathrm{pg} / \mathrm{mL}$ difference in the DPP-4 inhibitor group with a standard deviation of $500 \mathrm{pg} / \mathrm{mL}$ between the sitagliptin-treated and no additional treatment groups, a total of 10 subjects would be required at a $5 \%$ significance level and $80 \%$ power.

Values of $\mathrm{p}<0.05$ (two-tailed) were considered statistically significant. All statistical analyses were performed using the SPSS software program, version 20 (SPSS, Chicago, USA).

\section{Results}

\section{Patient characteristics}

Seventy-six patients with CAD were initially enrolled in this study. Forty-six patients were excluded based on the exclusion criteria, blood samples, or flow wire evaluations that were either inappropriate for measurement or lacked sufficient follow-up coronary angiography findings, including a flow wire study.

Table 1 shows the baseline demographics, medications, and glycemic control of subjects enrolled in the study. At baseline, the DPP-4 inhibitor group had a significantly higher HbA1c and lower 1,5-AG than the standard therapy group. During follow-up, no hospitalizations for heart failure, cardiovascular death, lethal ventricular tachycardia or 
Table 1. Patient Characteristics.

\begin{tabular}{|c|c|c|c|c|}
\hline Variables & $\begin{array}{l}\text { Total patients } \\
\quad(\mathrm{n}=30)\end{array}$ & $\begin{array}{l}\text { DPP-4 inhibitor on } \\
\qquad(\mathrm{n}=11)\end{array}$ & $\begin{array}{l}\text { DPP-4 inhibitor off } \\
\qquad(\mathrm{n}=19)\end{array}$ & $\mathrm{p}$ \\
\hline Age, years & $72.0[67.7-76.7]$ & $72.0[64.0-79.0]$ & $71.0[69.0-73.0]$ & 0.67 \\
\hline $\operatorname{Sex}(M / F), n(\%)$ & $23(77) / 7(23)$ & $8(73) / 3(27)$ & $15(79) / 4(21)$ & $>0.99$ \\
\hline Hypertension, n (\%) & $26(87)$ & $9(82)$ & $17(89)$ & 0.61 \\
\hline $\mathrm{DM}, \mathrm{n}(\%)$ & $30(100)$ & $11(100)$ & $19(100)$ & $>0.99$ \\
\hline DL, n (\%) & $24(80)$ & $9(82)$ & $15(79)$ & $>0.99$ \\
\hline ARB/ACE-I, n (\%) & $22(73)$ & $8(73)$ & $14(74)$ & $>0.99$ \\
\hline Statin, n $(\%)$ & $22(73)$ & $8(73)$ & $14(74)$ & $>0.99$ \\
\hline Insulin, $\mathrm{n}(\%)$ & $3(10)$ & $0(0)$ & $3(16)$ & 0.27 \\
\hline Biguanide, n (\%) & $2(7)$ & $2(18)$ & $0(0)$ & 0.20 \\
\hline Sulfonylurea, n (\%) & $6(20)$ & $5(45)$ & $1(5)$ & 0.01 \\
\hline Alpha-GI, n (\%) & $4(13)$ & $2(18)$ & $2(11)$ & 0.61 \\
\hline
\end{tabular}

ACE-I: angiotensin-converting enzyme inhibitor, Alpha-GI: alpha-glucosidase inhibitor, ARB: angiotensin II receptor blocker, DL: dyslipidemia, DM: diabetes mellitus, DPP-4: dipeptidyl peptidase-4

Continuous values are expressed as the median (interquartile range).
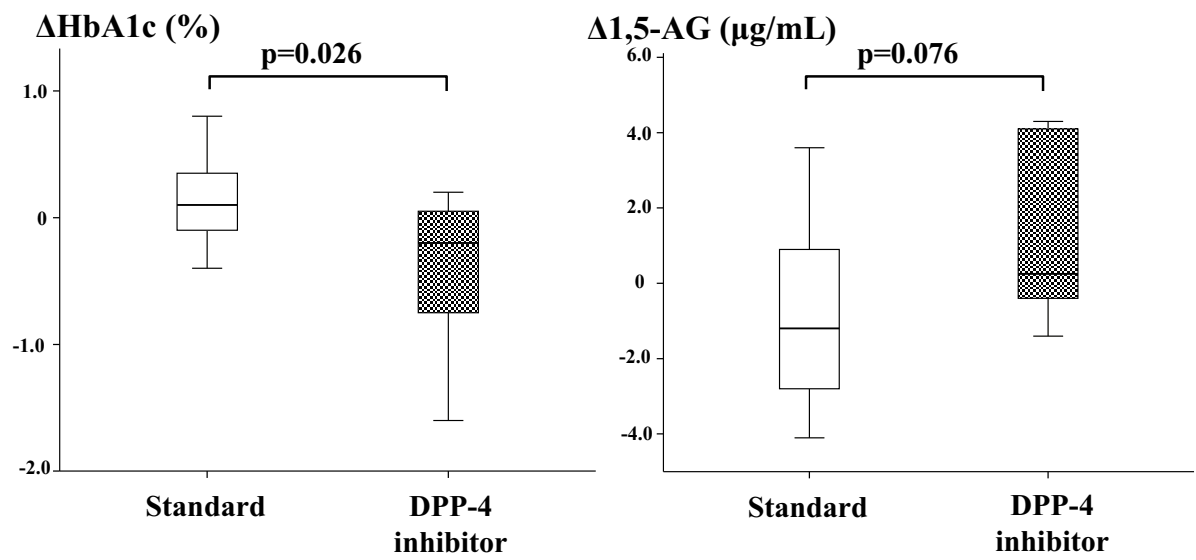

Figure 1. Bar graphs illustrating changes in the HbA1c and 1,5-AG levels in CAD patients with DM between baseline and the post-24-week therapeutic period. The horizontal line represents the median, the box encompasses the 25th to 75th percentiles, and the error bars encompass the 10th to 90th percentiles. $P$ values were obtained by comparing values with and without DPP-4 inhibitor. 1,5-AG: 1,5-anydroglucitol, DPP-4: dipeptidyl peptidase-4

ventricular fibrillation, or urgent revascularization were encountered.

\section{Effects of sitagliptin on HbA1c and 1,5-AG}

Figure 1 shows the effects of sitagliptin on glycemic control, as represented by the $\mathrm{HbA1c}$ and 1,5-AG. Sitagliptin therapy improved the HbA1c and 1,5-AG markedly in comparison with the standard therapy (HbA1c, $-0.4 \pm 0.8$ vs. 0.1 $\pm 0.3 \%, \mathrm{p}=0.02$; $1,5-\mathrm{AG}, 5.3 \pm 5.2$ vs. $-0.8 \pm 2.6 \mu \mathrm{g} / \mathrm{mL}, \mathrm{p}=$ $0.07)$.

\section{Effects of sitagliptin on BNP and E/e'}

At baseline, no significant differences were observed in the BNP or E/e' values between the DPP-4 inhibitor group and standard therapy group (Table 2). Patients receiving a DPP-4 inhibitor showed neither significant increases nor decreases in the BNP or E/e' compared with the ordinary regimen subjects after 24 weeks, but they did show improve- ment in the 1.5-AG and HbA1c values compared with the standard therapy group (Fig. 2). No differences were evident between the 2 groups in terms of changes in the BNP (DPP-4 inhibitor, $7.1 \pm 24.5$ vs. standard therapy, $-23.0 \pm 22.7$ $\mathrm{pg} / \mathrm{mL} ; \mathrm{p}=0.22)$ or $\mathrm{E} / \mathrm{e}$ ' $(-0.1 \pm 3.3$ vs. $-0.7 \pm 2.6 ; \mathrm{p}=0.58)$ (Fig. 2). The mean change from baseline in the EF did not differ significantly between the DPP-4 inhibitor group and the standard therapy group $(-6.0 \pm 9.6$ and $3.1 \pm 11.2 \%, \mathrm{p}=$ 0.06).

\section{Effects of sitagliptin on CFR and ADMA}

At baseline, no significant differences were observed in the CFR or ADMA between the DPP-4 inhibitor group and the standard therapy groups (Table 2), although both groups included a considerable proportion of patients with coronary microvascular dysfunction (6/11, 54\%; and 11/19, 57\%). With regard to coronary and systemic vascular endothelial dysfunction, serial changes in the CFR and ADMA did not 
Table 2. Clinical Parameters at Baseline and Follow-up.

\begin{tabular}{llccc}
\hline \multirow{2}{*}{ Variable } & & $\begin{array}{c}\text { DPP-4 inhibitor on } \\
(\mathrm{n}=11)\end{array}$ & $\begin{array}{c}\text { DPP-4 inhibitor off } \\
(\mathrm{n}=19)\end{array}$ & $\mathrm{p}$ \\
\hline $\mathrm{HbA1c}, \%(\mathrm{mmol} / \mathrm{mol})$ & Baseline & $7.3[6.3-7.7][56(45-61)]$ & $5.8[5.4-6.8][40(36-51)]$ & 0.006 \\
& Follow-up & $6.4[6.2-7.5][46(44-58)]$ & $5.8[5.6-7.1][40(38-54)]$ & 0.11 \\
$1,5-\mathrm{AG}, \mu \mathrm{g} / \mathrm{mL}$ & Baseline & $6.8[5.2-14.8]$ & $20.3[15.0-22.7]$ & 0.008 \\
& Follow-up & $8.5[6.0-16.7]$ & $16.9[12.9-26.3]$ & 0.06 \\
$\mathrm{CFR}$ & Baseline & $1.9[1.6-2.8]$ & $1.9[1.5-2.2]$ & 0.47 \\
& Follow-up & $1.6[1.4-2.0]$ & $1.9[1.3-2.3]$ & 0.63 \\
$\mathrm{BMI}, \mathrm{kg} / \mathrm{m}^{2}$ & Baseline & $25.4[22.4-27.8]$ & $23.0[21.1-27.4]$ & 0.23 \\
& Follow-up & $22.5[21.2-26.7]$ & $24.6[22.7-27.0]$ & 0.18 \\
$\mathrm{LDL}, \mathrm{mg} / \mathrm{dL}$ & Baseline & $85.0[69.0-117.0]$ & $82.0[72.0-116.0]$ & 0.86 \\
& Follow-up & $81.0[61.0-106.0]$ & $98.5[73.2-114.0]$ & 0.31 \\
$\mathrm{EF}, \%$ & Baseline & $64.8[61.6-76.1]$ & $64.2[52.1-71.4]$ & 0.61 \\
$\mathrm{E} / \mathrm{E} \#$ & Follow-up & $59.5[55.6-63.9]$ & $63.7[57.5-72.0]$ & 0.37 \\
& Baseline & $12.6[10.5-15.9]$ & $13.2[10.4-15.5]$ & 0.96 \\
$\mathrm{BNP}, \mathrm{pg} / \mathrm{mL}$ & Follow-up & $13.9[10.8-17.3]$ & $12.9[9.0-15.4]$ & 0.35 \\
& Baseline & $16.9[10.2-51.6]$ & $39.2[12.0-96.1]$ & 0.45 \\
$\mathrm{SDF}-1 \alpha, \mathrm{pg} / \mathrm{mL}$ & Follow-up & $20.2[14.7-47.3]$ & $28.7[7.3-76.1]$ & 0.87 \\
& Baseline & $1,451.9[1,189.4-2,378.9]$ & $1,908.0[1,579.0-2,319.2]$ & 0.14 \\
$\mathrm{EPC}, \%$ & Follow-up & $1,044.6[902.6-1,210.3]$ & $1,720.6[1,387.8-2,016.7]$ & $<0.001$ \\
& Baseline & $0.00[0.00-0.32]$ & $0.00[0.00-0.40]$ & 0.96 \\
& Follow-up & $0.00[0.00-0.00]$ & $0.00[0.00-0.06]$ & 0.73 \\
\hline
\end{tabular}

1,5-AG: 1,5-anhydroglucitol, BMI: body mass index, BNP: brain natriuretic peptide, CFR: coronary flow reserve, DPP4: dipeptidyl peptidase-4, EF: ejection fraction, EPC: endothelial progenitor cell, LDL: low-density lipoprotein, SDF$1 \alpha$ : stromal cell-derived factor- 1 alpha

Continuous values are expressed as the median (interquartile range).
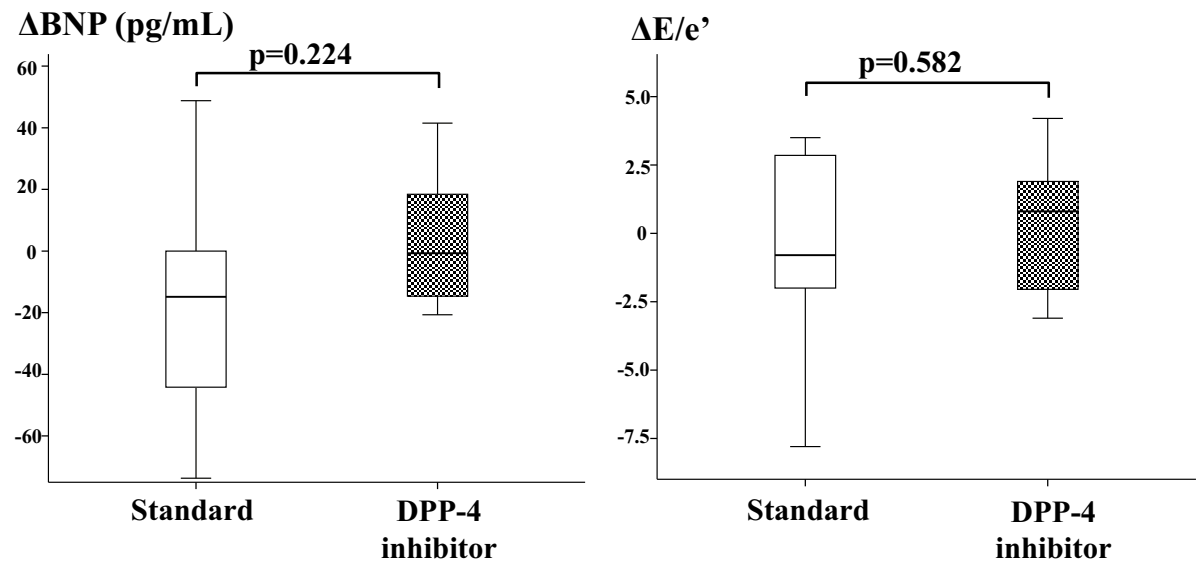

Figure 2. Bar graphs illustrating changes in BNP and E/e' levels in CAD patients with DM between baseline and the post-24-week therapeutic period. The horizontal line represents the median, the box encompasses the 25th to 75th percentiles, and the error bars encompass the 10th to 90th percentiles. $P$ values were obtained by comparing values with and without DPP-4 inhibitor. BNP: brain natriuretic peptide, DPP-4: dipeptidyl peptidase-4

differ markedly between the two groups (Fig. 3). After 24 weeks, compared with standard therapy subjects, patients receiving a DPP-4 inhibitor did not show significant increases in the CFR $(0.0 \pm 0.2$ vs. $0.1 \pm 0.6, p=0.20)$ or ADMA $(-0.39 \pm$ 0.64 vs. $0.21 \pm 0.25 \mu \mathrm{mol} / \mathrm{L}, \mathrm{p}=0.14)$.

\section{Effects of sitagliptin on the EPC count and SDF-1 levels}

At baseline, no significant differences were observed in the EPC proportion or SDF-1 $\alpha$ levels between the DPP-4 inhibitor and standard therapy groups (Table 2). After 24 weeks, compared with ordinary regimen subjects, patients receiving a DPP-4 inhibitor did not show significant in- 

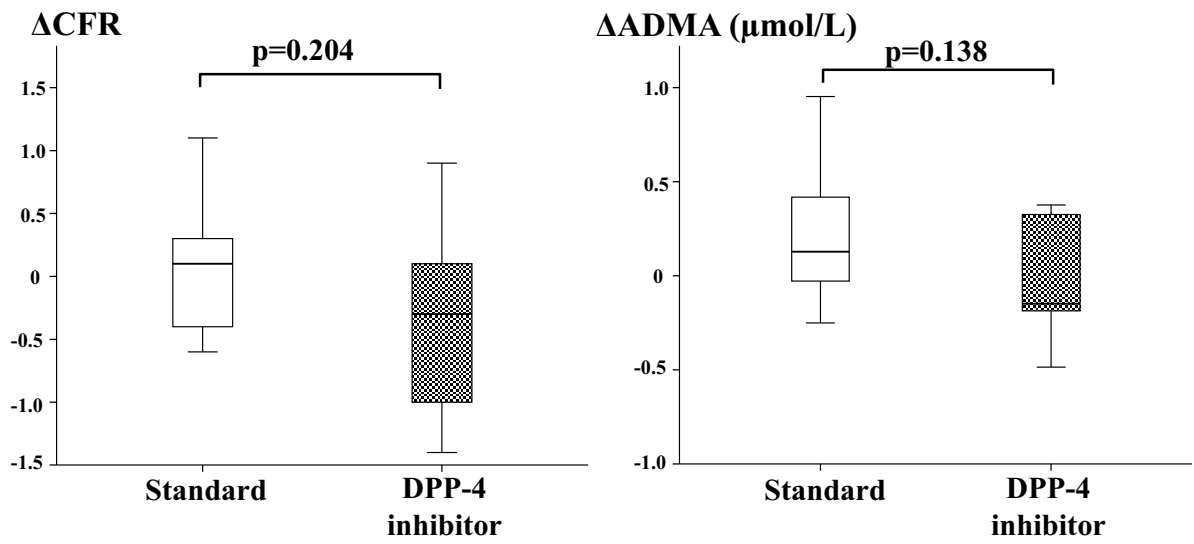

Figure 3. Bar graphs illustrating changes in the CFR and ADMA levels for CAD patients with DM between baseline and the post-24-week therapeutic period. The horizontal line represents the median, the box encompasses the 25th to 75th percentiles, and the error bars encompass the 10th to 90th percentiles. $P$ values were obtained by comparing values with and without DPP-4 inhibitor. ADMA: asymmetric dimethylarginine, CFR: coronary flow reserve, DPP-4: dipeptidyl peptidase-4
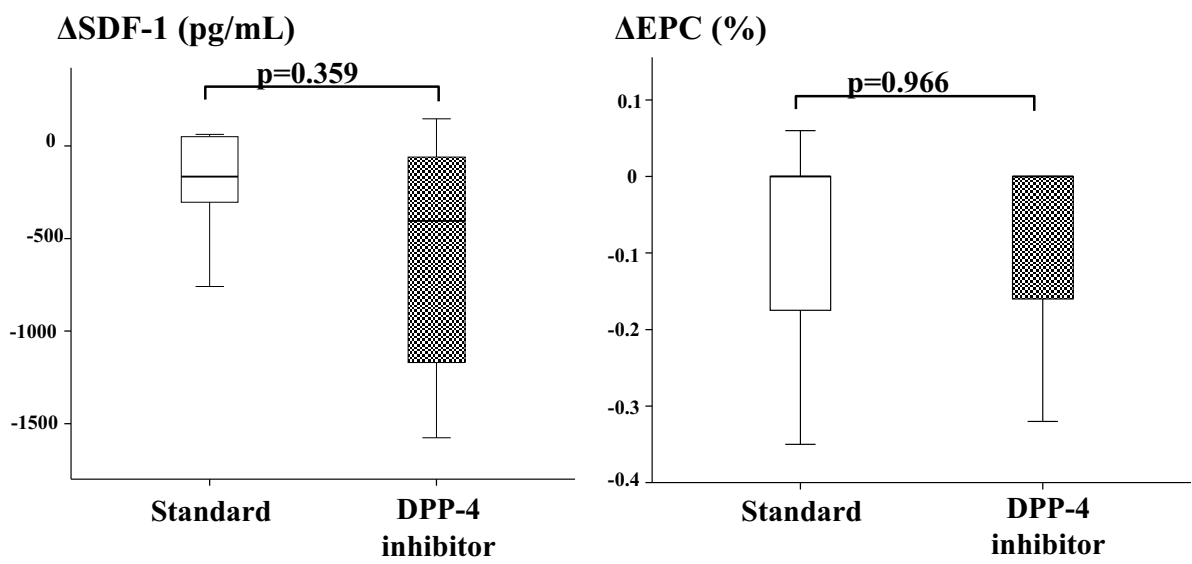

Figure 4. Bar graphs illustrating changes in the SDF-1 $\alpha$ levels and EPC counts in CAD patients with DM between baseline and the post-24-week therapeutic period. The horizontal line represents the median, the box encompasses the 25th to 75 th percentiles, and the error bars encompass the 10th to 90th percentiles. $P$ values were obtained by comparing values with and without DPP-4 inhibitor. SDF-1 $\alpha$ : stromal cell-derived factor-1alpha, DPP-4: dipeptidyl peptidase-4, EPC: endothelial progenitor cell

creases in EPC proportions $(-0.20 \pm 0.25$ vs. $0.24 \pm 1.11 \%, \mathrm{p}=$ $0.96)$ or SDF- $1 \alpha$ levels $(-888.9 \pm 585.2$ vs. $-311.8 \pm 476.4 \mathrm{pg} /$ $\mathrm{mL}, \mathrm{p}=0.35)$. No significant correlation was noted between changes in the SDF-1 $\alpha$ levels or EPC proportion ( $r s<0.01$, $\mathrm{p}=0.99)($ Fig. 4).

\section{Predictors of $\triangle E P C$}

There was no significant correlation between the $\triangle \mathrm{EPC}$ and other serial changes in data $(\Delta \mathrm{HbA} 1 \mathrm{c}, \Delta 1,5-\mathrm{AG}$, and $\triangle$ SDF-1). A multiple linear regression analysis was performed to identify predictors of $\triangle \mathrm{EPC}$. The interaction of covariates (age, sex, $\Delta \mathrm{BMI}, \Delta \mathrm{SDF}-1, \Delta 1,5-\mathrm{AG}$, and $\Delta \mathrm{HbA} 1 \mathrm{c})$ was evaluated using a multivariate linear regression model of $\triangle \mathrm{EPC}$. Another model of a multivariate linear regression analysis evaluated the interactions of baseline covariates (age, gender, HbAlc, 1,5-AG, EF, BMI, BNP and administration of DPP-4 inhibitor) for the determinant of $\triangle$ EPC. Covariates were used together in the multivariate linear regression analysis. As a result, no significant predictors or determinants were found in either analysis model.

\section{Discussion}

\section{Major findings}

This study demonstrated the following findings: First, the administration of sitagliptin improved glycated hemoglobin and 1,5-AG levels. Second, the addition of sitagliptin did not compromise the BNP levels or diastolic function, as reflected by E/e'. Third, short-term sitagliptin therapy did not improve the coronary flow or systemic endothelial function, as reflected by the CFR and ADMA, in comparison with or- 
dinary anti-DM therapy in CAD patients. Fourth, no intergroup differences were evident in changes to EPC counts or SDF-1 $\alpha$ levels.

These findings indicate that 1) sitagliptin therapy improved glycemic control without compromising the BNP levels or E/e', and 2) the effects on the coronary flow and endothelial function were comparable to those of standard anti-DM agents at 24 weeks.

\section{Efficacy of DPP-4 inhibitor therapy in patients with $D M$ and CAD}

There has been considerable speculation that DPP-4 inhibitors may exert beneficial effects on the cardiovascular system. However, the SAVOR-TIMI 53 study reported that the rate of hospitalization for heart failure was increased with this therapy (17), although our study did not find that DPP-4 inhibitor therapy was associated with any changes in the BNP level or diastolic function, as assessed by E/e' values. No significant increases in the BNP levels or E/e' were observed among patients in the DPP-4 inhibitor group compared with the standard therapy group.

In the subanalysis of the SAVOR-TIMI53 trial (28), baseline risk factors were found to be associated with an increased risk of hospitalization for heart failure, particularly in patients with a high absolute risk of heart failure. The factors strongly associated with hospitalization for heart failure were the history of heart failure, baseline estimated glomerular filtration rate, and albumin/creatinine ratio. There were no evidence of any interaction between the levels of N-terminal-proBNP, saxagliptin treatment, and hospitalization for heart failure in that subanalysis. In addition, there were no signs of a worsening cardiac diastolic function in our study with DPP-4 inhibitor, nor did sitagliptin increase the levels of BNP. The results of the SAVOR-TIMI53 trial may have been obtained purely by chance (random-high).

\section{Clinical implications}

In this prospective, parallel-design study, sitagliptin therapy did not increase the EPC counts or SDF- $1 \alpha$ levels or impair the cardiac function when added to standard treatment with anti-diabetic agents in patients with CAD.

An apparent discrepancy between the findings of in vitro studies $(8,29)$ and our results was observed, with several potentially viable explanations. First, the exposure to sitagliptin may not have been long enough to reverse the effects of advanced atherosclerotic processes in patients with CAD. For example, the United Kingdom Prospective Diabetes (UKPD) study required a relatively long follow-up period to confirm a beneficial effect of glycemic control (30). Our study had a duration of 24 weeks; the results therefore do not rule out potential longer-term benefits or risks of DPP-4 inhibitors with respect to cardiovascular effects. Alternatively, the therapeutic period of 24 weeks may have been too long. With this relatively long duration of therapy, underlying risk factors and atherosclerotic burden may have diminished the clinical benefits of the DPP-4 inhibitor. Our study population already has an advanced atherosclerotic status (Table 1), so the benefits of DPP-4 inhibitor may have been offset by underlying risk factors. In patients with DM, EPC proportions are reduced, and EPCs show a reduced recruitment from bone marrow to the peripheral vasculature (31). In addition, the rate of apoptosis also influences the number of EPCs (32), but markers of apoptosis were not measured in our study. We were therefore unable to elucidate the significant association between EPC numbers and apoptosis. Second, the glycated hemoglobin levels differed between the study groups. Furthermore, a large proportion of patients in our study had several comorbidities, such as dyslipidemia and hypertension. This complicated background may have blunted any potential differences between study groups. The current standard of anti-DM therapy is not sufficient to address overt progressing atherosclerosis. Patients with intolerance on glucose testing are candidates for interventional therapy to overcome subsequent worsening of endothelial dysfunction $(33,34)$. Third, the therapeutic dose may not have been sufficient to obtain any additional clinical effect. In an in vitro study, the administration of DPP-4 inhibitor increased SDF- $1 \alpha$ levels and the EPC proportion (8), but the clinical doses in the present study may have been too low to obtain sufficient upregulation of SDF$1 \alpha$ and EPCs in vivo.

We hypothesized that DPP-4 inhibition inactivated the DPP-4 ability to cleave SDF- $1 \alpha$, leading to increased EPC counts via the enhancement of SDF- $1 \alpha$ levels. SDF- $1 \alpha$ is a potent chemoattractant for EPCs $(5,7)$. The role of SDF- $1 \alpha$ in angiogenesis and the exact mechanism underlying SDF$1 \alpha$ and vasculogenesis remains to be elucidated (6). In animal and molecular models, DPP-4 inhibitor promotes angiogenesis by the SDF- $1 \alpha$-dependent mobilization of EPCs with homing to ischemic tissues. However, the results of the current study conflicted with our hypothesis. One potential reason for the unexpected findings in our study is the involvement of other exopeptidases that cleave SDF- $1 \alpha$ as a substrate. Indeed, SDF- $1 \alpha$ is also sensitive to other exopeptidases, such as matrix metalloproteinase (MMP) family members (MMP-2, MMP-9) and other DPP families (DPP8 , DPP-9) $(29,35,36)$. In the present study, the baseline DM status was not equivalent between the two groups. This may explain the difference in the SDF- $1 \alpha$ level between the groups in our study. The DPP-4 inhibitor group may have had a more severe atherosclerotic condition at the baseline measurement than the control group. Under advanced atherosclerotic conditions, as in cases of CAD and DM vasculopathy, inflammatory cytokines, such as MMP-2 and MMP-9, interleukin-6, tumor necrosis factor-alpha, C-reactive protein, and DPP-8 and DPP-9, are upregulated. Thus, differences in the SDF- $1 \alpha$ levels at baseline between the two treatment groups may have been due to compensatory increases in the peripheral SDF-1 $\alpha$ degradation enzymes, such as MMP-2 and MMP-9 and DPP-8 and DPP-9. DPP-4 is not a unique SDF- $1 \alpha$ degradation enzyme. Therefore, merely suppressing the DPP-4 activity may not always increase the SDF- $1 \alpha$ 
level in peripheral blood.

Concern has been raised about a possible association between DPP-4 inhibitor therapy and an increased risk of heart failure (17). Our study found that changes in the BNP and E/e' values did not differ markedly between groups. These results are consistent with previous findings concerning the DPP-4 inhibitor effect on cytokines and the diastolic function $(10,37)$. In addition, our observation that DPP-4 inhibitor therapy was not associated with changes in the BNP level or E/e' was consistent with the findings from the TECOS clinical trials (38). Our study showed that the addition of a DPP-4 inhibitor to standard diabetic care did not have significant effects on the cardiac function.

\section{Limitations}

Several limitations associated with the present study warrant mention. First, we enrolled patients with DM and CAD, but the baseline glycated hemoglobin and 1,5-AG levels differed between the DPP-4 inhibitor therapy group and the standard therapy group. Potential biases include possible confounding factors of residual intergroup differences in the glycated hemoglobin level and the greater use of antidiabetic agents in the DPP-4 inhibitor therapy group than in the standard therapy group. For instance, biguanides are the most commonly prescribed antidiabetic agent in CAD patients as first-line drugs (39). However, in the present study, only 2 out of 30 patients were prescribed biguanides. The risk of lactic acidosis after the administration of contrast medium for patients receiving biguanides is widely known. In our study protocol, follow-up coronary angiography was scheduled. Thus, the attending physicians may have avoided the use of biguanides. To investigate the differing effects of DPP-4 inhibitors between these two groups, we need to minimize and offset the differences in their baseline characteristics. This can be achieved by performing analyses with three approaches to statistical adjustment: (1) compare the changes from the baseline to follow-up $(\Delta)$; (2) assess the correlation between the $\Delta$ value and the $\Delta \mathrm{EPC}$; (3) perform a multivariate linear regression analysis to identify the determinant of $\triangle \mathrm{EPC}$, including covariates of background characteristics. However, there were significant differences between the DPP-4 inhibitor group and the standard therapy group according to the baseline characteristics. Therefore, the effect of DPP-4 treatment might be offset by these differences even after statistical adjustments.

Second, we evaluated the SDF- $1 \alpha$ levels in the present study, but the levels of SDF- $1 \alpha$ are regulated by DPP-4 activity as well as vascular endothelial factor (5) and MMP2 (29), so their influence cannot be discounted. We tested whether or not the regulation of SDF- $1 \alpha$ by DPP-4 treatment is involved in improving the CFR via EPCs in this study. An analysis of the whole-cytokine/chemokine interactions may lead to a better understanding of the continuum mechanisms controlling SDF- $1 \alpha$ concentrations.

Third, the numbers of EPCs and participants in this analysis were relatively small. Further prospective studies with larger sample sizes, longer follow-up, and serial measurements of a variety of biomarkers and clinical parameters over a longer period may therefore be needed in order to resolve these limitations and validate our results.

DPP-4 inhibitor therapy for 24 weeks improved glycemic control without worsening the EPC counts, CFR, or SDF- $1 \alpha$ levels in $\mathrm{CAD}$ patients compared with patients on ordinary anti-DM therapy. However, additional clinical evidence for the upregulation of EPCs via SDF- $1 \alpha$ levels and the CFR was not observed. Our study was unable to determine the beneficial effects of DPP-4 inhibitors on the CFR, EPC count, or SDF-1 levels. Further studies are needed to confirm our results.

Among patients with $\mathrm{DM}$ and $\mathrm{CAD}$, treatment with a DPP-4 inhibitor resulted in changes to the EPC counts, SDF-1 $\alpha$ and BNP levels, and E/e' that were similar to those obtained with standard anti-diabetic therapy, along with considerable improvement of glycated hemoglobin and postprandial hyperglycemia.

\section{The authors state that they have no Conflict of Interest (COI).}

\section{Acknowledgement}

We wish to thank Mses. Hiromi Nishimura, Yumie Yasusaki, Motoko Oku, Mari Kurata, and Yoshiko Kurose for providing excellent technical assistance. The first author would also like to express his gratitude to his wife and daughter for their moral support and constant encouragement.

\section{References}

1. Asahara T, Murohara T, Sullivan A, et al. Isolation of putative progenitor endothelial cells for angiogenesis. Science 275: 964-967, 1997.

2. Nakamura Y, Suzuki S, Shimizu T, et al. High mobility group box 1 promotes angiogenesis from bone marrow-derived endothelial progenitor cells after myocardial infarction. J Atheroscler Thromb 22: 570-581, 2015.

3. Hirase T. Capturing VE-cadherin-positive endothelial progenitor cells for in-stent vascular repair. J Atheroscler Thromb 23: 46-47, 2016.

4. Fadini GP, Sartore S, Albiero M, et al. Number and function of endothelial progenitor cells as a marker of severity for diabetic vasculopathy. Arterioscler Thromb Vasc Biol 26: 2140-2146, 2006.

5. Tang JM, Wang JN, Zhang L, et al. VEGF/SDF-1 $\alpha$ promotes cardiac stem cell mobilization and myocardial repair in the infarcted heart. Cardiovasc Res 91: 402-411, 2011.

6. Abbott JD, Huang Y, Liu D, Hickey R, Krause DS, Giordano FJ. Stromal cell-derived factor-1alpha plays a critical role in stem cell recruitment to the heart after myocardial infarction but is not sufficient to induce homing in the absence of injury. Circulation 110: 3300-3305, 2004.

7. Yamaguchi J, Kusano KF, Masuo O, et al. Stromal cell-derived factor-1 effects on ex vivo expanded endothelial progenitor cell recruitment for ischemic neovascularization. Circulation 107: 13221328, 2003.

8. Proost P, Struyf S, Schols D, et al. Processing by CD26/ dipeptidyl-peptidase IV reduces the chemotactic and anti-HIV-1 activity of stromal-cell-derived factor-1alpha. FEBS Lett 432: 7376, 1998.

9. Fadini GP, Boscaro E, Albiero M, et al. The oral dipeptidyl 
peptidase-4 inhibitor sitagliptin increases circulating endothelial progenitor cells in patients with type 2 diabetes: possible role of stromal-derived factor-1alpha. Diabetes Care 33: 1607-1609, 2010.

10. Ayaori M, Iwakami N, Uto-Kondo H, et al. Dipeptidyl peptidase-4 inhibitors attenuate endothelial function as evaluated by flowmediated vasodilatation in type 2 diabetic patients. J Am Heart Assoc 2: e003277, 2013.

11. Bando YK, Murohara T. Heart failure as a comorbidity of diabetes: role of dipeptidyl peptidase 4. J Atheroscler Thromb 23: 147154, 2016.

12. Kohashi K, Hiromura M, Mori Y, et al. A dipeptidyl peptidase-4 inhibitor but not incretins suppresses abdominal aortic aneurysms in angiotensin II-infused apolipoprotein E-null mice. J Atheroscler Thromb 23: 441-454, 2016.

13. Nozue T, Fukui K, Takamura T, et al. Effects of alogliptin on fractional flow reserve evaluated by coronary computed tomography angiography in patients with type 2 diabetes: rationale and design of the TRACT study. J Cardiol 69: 518-522, 2017.

14. Takashio S, Yamamuro M, Izumiya Y, et al. Coronary microvascular dysfunction and diastolic load correlate with cardiac troponin T release measured by a highly sensitive assay in patients with nonischemic heart failure. J Am Coll Cardiol 62: 632-640, 2013.

15. Böger RH, Bode-Böger SM, Szuba A, et al. Asymmetric dimethylarginine (ADMA): a novel risk factor for endothelial dysfunction: its role in hypercholesterolemia. Circulation 98: 1842-1847, 1998.

16. Kielstein JT, Impraim B, Simmel S, et al. Cardiovascular effects of systemic nitric oxide synthase inhibition with asymmetrical dimethylarginine in humans. Circulation 109: 172-177, 2004.

17. Scirica BM, Bhatt DL, Braunwald E, et al. Saxagliptin and cardiovascular outcomes in patients with type 2 diabetes mellitus. $\mathrm{N}$ Engl J Med 369: 1317-1326, 2013.

18. Watanabe $\mathrm{K}$, Uchino $\mathrm{H}$, Ohmura $\mathrm{C}$, Tanaka $\mathrm{Y}$, Onuma $\mathrm{T}$, Kawamori R. Different effects of two alpha-glucosidase inhibitors, acarbose and voglibose, on serum 1,5-anhydroglucitol (1,5AG) level. J Diabetes Complications 18: 183-186, 2004.

19. Morishita T, Uzui H, Ikeda H, et al. Association of CD34/CD133/ VEGFR2-positive cell numbers with eicosapentaenoic acid and postprandial hyperglycemia in patients with coronary artery disease. Int J Cardiol 221: 1039-1042, 2016.

20. Morishita T, Uzui H, Nakano A, et al. Number of endothelial progenitor cells in peripheral artery disease as a marker of severity and association with pentraxin-3, malondialdehyde-modified lowdensity lipoprotein and membrane type-1 matrix metalloproteinase. J Atheroscler Thromb 19: 149-158, 2012.

21. Watanabe M, Kokubo Y, Higashiyama A, Ono Y, Miyamoto Y, Okamura T. Serum 1,5-anhydro-D-glucitol levels predict first-ever cardiovascular disease: an 11-year population-based cohort study in Japan, the Suita study. Atherosclerosis 216: 477-483, 2011.

22. Ouchi M, Oba K, Motoyama M, et al. Postprandial glycemic control conditions in relation to urinary $\mathrm{N}$-acetyl- $\beta$-D-glucosaminidase in patients with type 2 diabetes mellitus without low glomerular filtration rate. Diabetes Technol Ther 16: 41-47, 2014.

23. Morishita T, Uzui H, Mitsuke $Y$, et al. Predictive utility of the changes in matrix metalloproteinase- 2 in the early phase for left ventricular reverse remodeling after an acute myocardial infarction. J Am Heart Assoc 4: e001359, 2015.

24. Lang RM, Bierig M, Devereux RB, et al. Recommendations for chamber quantification: a report from the American Society of Echocardiography's Guidelines and Standards Committee and the Chamber Quantification Writing Group, developed in conjunction with the European Association of Echocardiography, a branch of the European Society of Cardiology. J Am Soc Echocardiogr 18:
1440-1463, 2005.

25. Sohn DW, Chai IH, Lee DJ, et al. Assessment of mitral annulus velocity by Doppler tissue imaging in the evaluation of left ventricular diastolic function. J Am Coll Cardiol 30: 474-480, 1997.

26. van de Hoef TP, van Lavieren MA, Damman P, et al. Physiological basis and long-term clinical outcome of discordance between fractional flow reserve and coronary flow velocity reserve in coronary stenoses of intermediate severity. Circ Cardiovasc Interv 7: 301-311, 2014.

27. Ikeda H, Uzui H, Morishita T, et al. Effect of postprandial hyperglycaemia on coronary flow reserve in patients with impaired glucose tolerance and type 2 diabetes mellitus. Diab Vasc Dis Res 12: 405-410, 2015.

28. Scirica BM, Braunwald E, Raz I, et al. Heart failure, saxagliptin, and diabetes mellitus: observations from the SAVOR-TIMI 53 randomized trial. Circulation 130: 1579-1588, 2014.

29. Kanki S, Segers VF, Wu W, et al. Stromal cell-derived factor-1 retention and cardioprotection for ischemic myocardium. Circ Heart Fail 4: 509-518, 2011.

30. Holman RR, Paul SK, Bethel MA, Matthews DR, Neil HA. 10year follow-up of intensive glucose control in type 2 diabetes. $\mathrm{N}$ Engl J Med 359: 1577-1589, 2008.

31. Fadini GP, Boscaro E, de Kreutzenberg S, et al. Time course and mechanisms of circulating progenitor cell reduction in the natural history of type 2 diabetes. Diabetes Care 33: 1097-1102, 2010.

32. Jung C, Rafnsson A, Shemyakin A, Böhm F, Pernow J. Different subpopulations of endothelial progenitor cells and circulating apoptotic progenitor cells in patients with vascular disease and diabetes. Int J Cardiol 143: 368-372, 2010.

33. Chiasson JL, Josse RG, Gomis R, et al. Acarbose treatment and the risk of cardiovascular disease and hypertension in patients with impaired glucose tolerance: the STOP-NIDDM trial. JAMA 290: 486-494, 2003.

34. Hanefeld M, Cagatay M, Petrowitsch T, Neuser D, Petzinna D, Rupp M. Acarbose reduces the risk for myocardial infarction in type 2 diabetic patients: meta-analysis of seven long-term studies. Eur Heart J 25: 10-16, 2004.

35. Askari AT, Unzek S, Popovic ZB, et al. Effect of stromal-cellderived factor 1 on stem-cell homing and tissue regeneration in ischaemic cardiomyopathy. Lancet 362: 697-703, 2003.

36. Sun CK, Leu S, Sheu JJ, et al. Paradoxical impairment of angiogenesis, endothelial function and circulating number of endothelial progenitor cells in DPP4-deficient rat after critical limb ischemia. Stem Cell Res Ther 4: 31, 2013.

37. Oe H, Nakamura K, Kihara H, et al. Comparison of effects of sitagliptin and voglibose on left ventricular diastolic dysfunction in patients with type 2 diabetes: results of the 3D trial. Cardiovasc Diabetol 14: 83, 2015.

38. Green JB, Bethel MA, Armstrong PW, et al. Effect of sitagliptin on cardiovascular outcomes in type 2 diabetes. N Engl J Med 373: 232-242, 2015.

39. Low Wang CC, Hess CN, Hiatt WR, Goldfine AB. Clinical update: cardiovascular disease in diabetes mellitus: atherosclerotic cardiovascular disease and heart failure in type 2 diabetes mellitus - mechanisms, management, and clinical considerations. Circulation 133: 2459-2502, 2016.

The Internal Medicine is an Open Access journal distributed under the Creative Commons Attribution-NonCommercial-NoDerivatives 4.0 International License. To view the details of this license, please visit (https://creativecommons.org/licenses/ by-nc-nd/4.0/).

(C) 2019 The Japanese Society of Internal Medicine

Intern Med 58: 2773-2781, 2019 